\title{
Heat Transfer Enhancement in Wavy Micro-Channels: Effect of Block Material
}

\author{
Justin Moon $^{1}$, J. Rafael Pacheco ${ }^{2}$, Arturo Pacheco-Vega ${ }^{2}$ \\ ${ }^{1}$ California State University, Los Angeles \\ Los Angeles, CA 90032, USA \\ jmoon19@calstatela.edu \\ ${ }^{2}$ SAP America Inc. \\ Tempe AZ 85281, USA \\ apacheco@calstatela.edu; rafael.pacheco@sap.com
}

\begin{abstract}
In this study, three-dimensional numerical simulations were performed to investigate heat transfer enhancement in multiharmonic micro-scale wavy channels. The goal is on extending the analysis of our previous work [1], which focused on the influence of channel surface-topography (modelled as multi-harmonic sinusoidal waves of square cross-sectional area) on the enhancing mechanisms, by considering a variety of conductive materials for the solid block that encloses the channel, and their impact on the heat transfer enhancement in these devices. The governing equations for an incompressible laminar flow and conjugate heat transfer are first built and then solved, for representative single- and harmonic-wavy-channel models from [1], with copper, aluminium and silicon as solid-block materials and different operating conditions, by the finite element technique. Solutions show that, regardless of the material, as wave amplitude and Reynolds number increase, so does the effectiveness of the device, and that the selection of specific material highly impacts the diffusion of heat in the solid, but it is negligible on the Nusselt number for the fluid. Finally, results of the performance factor $P F$, indicate that the device is ineffective in the heat transfer enhancement relative to the required pumping power for small values of Reynolds number $R e$, but the device becomes very effective for larger $R e$-values.
\end{abstract}

Keywords: Wavy micro-channel; Harmonic surfaces; Numerical analysis; Conjugate heat transfer.

\section{Introduction}

The development of high power-density electronic devices have increased the need for larger heat removal capabilities, which may inhibit further advancement in the miniaturization of integrated circuits. A solution to the aforementioned problem was developed by Tuckerman and Pease [2] who proposed the use of micro-channels for high heat-flux applications. Since then, experimental and numerical studies of a variety of geometric designs of micro-channel devices [3]-[7], using a single-phase fluid, have demonstrated the potential to passively enhance the transfer of energy in electronic devices. A particular case is that of wavy geometries in micro-channels, aimed at enhancing heat transfer performance due to the improvement in mixing of the fluid. Although some studies have corroborated slightly-larger pressure drop along the channel, the benefit has been a substantial heat transfer enhancement [8]-[10]. However, deterioration of the thermal performance was unavoidable under some geometrical configurations and low Reynolds numbers. To deal with this problem, a number of works have studied different channel layouts [11]-[13], all limited to a single-wavelength wavy channel, representing the entire heat sink.

Preliminary work of heat transfer enhancement in microchannels based on multi-harmonic surface topologies has been recently reported by Moon et al. [1]. The focus was on the influence of channel surface topography on the enhancing mechanisms. Their results demonstrated that the addition of harmonic surfaces to the basic topology provides higher Nusselt number ratios, thus corroborating a larger heat transfer in the device. For the microchannel model consisting of water as the working fluid and copper as the solid material, the analysis using input parameters of Reynolds number $R e$, amplitude $A$, and harmonic number $n$, established the highest Nusselt number ratio for harmonic numbers $n= \pm 2$. However, a decrease in the efficiency of the device was also found, due to the coupling of the pressure drop in the so-called performance factor. 
In the present study, we extend the analysis by Moon et al. [1] on the conjugate heat transfer characteristics in multiharmonic wavy channels, by considering other conductive materials, besides copper, for the solid block and their impact on the heat transfer enhancement in these devices. Preliminary analysis of the impact of materials such as aluminium and silicon

in straight micro-channel arrays have been recently reported $[14,15]$. To this end, we first select representative single and harmonic-wavy-channel models from [1], with different solid block materials, and then build the corresponding conjugate heat transfer model. Later, the model equations are solved using the finite element method, to obtain velocity-, pressure- and temperature-fields for the fluid in the microchannel, and the temperature distribution in the corresponding solid block. Finally, values of the pressure drop, inlet-to-outlet fluid and wall temperatures, Nusselt number and performance factor are computed determined for the devices with the different block materials, to assess the relative system performance.

\section{Problem Description and Governing Equations}

The micro-channel device under consideration is illustrated schematically in Fig. 1(a) for a single-wave-type geometry, and in Fig. 1(b) for cases of multi-harmonic wall profiles. It corresponds to a typical cooling system of a circuit-energy-dissipating equipment. The model consists of a square cross-section channel that is enclosed by a solid block of a conducting material. The channel has dimensions of $500 \mu \mathrm{m}$ by $500 \mu \mathrm{m}$ by $20 \mathrm{~mm}$, with two $2 \mathrm{~mm}$ inlet- and outlet-straight sections and a $16 \mathrm{~mm}$ middle wavy section, as shown in Fig. 1. In our previous work [1], the material chosen for the solid block was copper $(\mathrm{Cu})$; here, we consider a set of two additional materials; i.e., aluminium $(A l)$, and silicon $(\mathrm{Si})$, to analyse the impact on the heat transfer enhancement in the wavy channel. The block has a shape of a square duct of thickness $1.5 \mathrm{~mm}$ and length $20 \mathrm{~mm}$. A heat flux of $47 \mathrm{~W} / \mathrm{cm}^{2}$ is uniformly applied at the bottom surface and is advected out of the system through the internal flow of the water in the channel.

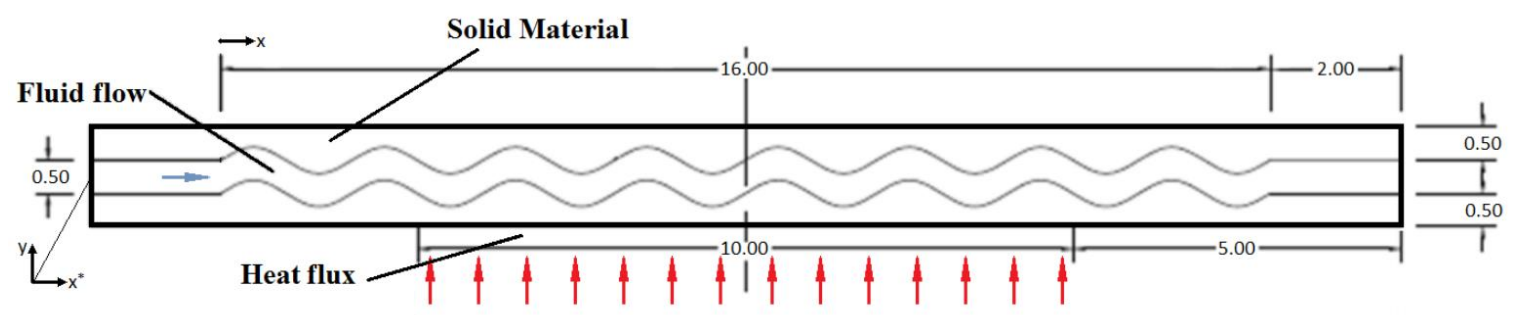

(a) Baseline geometry with dimensions in $\mathrm{mm}$.

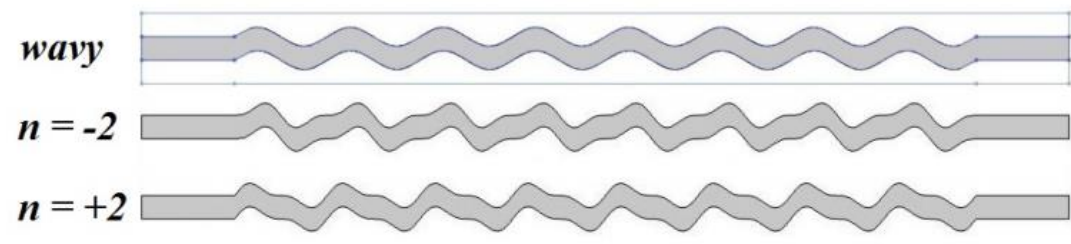

(b) Baseline and harmonic wall profiles.

Fig. 1: Side-view schematic of a wavy micro-channel.

The types of micro-channel-block designs herein studied are referred to as either wavy- (or baseline-), or multiharmonic-configuration. In the case of the wavy geometry, illustrated in Fig. 1(a), within the sinusoidal region the top and bottom surfaces are based on the function

$$
y(x)=A \cdot \sin (2 \pi x / \lambda)
$$


where $x$ and $y$ refer to the streamwise and vertical directions, respectively, $A$ as the wave amplitude and $\lambda$ is its wavelength. In the case of the multi-harmonic channel configuration, two cases from [1], that are illustrated in Fig. 1(b), are the $n= \pm 2$ values which modify the function

$$
y(x)=A \cdot \sin (2 \pi x / \lambda)+\frac{A}{|n|} \cdot \sin (2 \pi n x / \lambda)
$$

defining the shape of the bottom and top surfaces in the channel. The second term is the secondary sinusoidal term with scaled amplitude and angular frequency. It is to be noted that the values of $n= \pm 2$ have been chosen here due to the fact that the corresponding models provide the best heat transfer enhancement results in our previous work [1].

The governing equations correspond to a three-dimensional conjugate model for two domains: the channel inside of which water flows $\Omega_{\mathrm{f}}$, and the solid block $\Omega_{\mathrm{s}}$. For $\Omega_{\mathrm{f}}$, the model consists of the continuity, linear-momentum and energy equations for a steady laminar flow with constant fluid properties, incompressible Newtonian fluid (water), and negligible radiative heat transfer, given as

$$
\begin{aligned}
\nabla \cdot \mathbf{u} & =0 \\
\rho_{f}(\mathbf{u} \cdot \nabla) \mathbf{u} & =-\nabla p+\mu \nabla^{2} \mathbf{u} \\
\rho_{f} \mathrm{c}_{p, f}(\mathbf{u} \cdot \nabla) T & =k_{f} \nabla^{2} T
\end{aligned}
$$

For $\Omega_{\mathrm{s}}$, the energy equation for a homogeneous solid material is given by

$$
k_{s} \nabla^{2} T_{s}=0
$$

where $\mathbf{u}$, in Eqs. (3)-(5), is the Cartesian velocity vector with components, $u, v$ and $w$, in the directions $x, y$, and $z$, respectively; $p$ is the fluid pressure, $\rho_{f}$ is its density, and $\mathrm{c}_{p, f}$ is the specific heat, $k_{f}$ the fluid thermal conductivity, $\mu$ is the dynamic viscosity and $T$ its temperature. For the solid block, $k_{s}$ and $T_{s}$, in Eq. (6), are the thermal conductivity of the block material and its corresponding temperature.

The boundary conditions include: a prescribed streamwise velocity $u=u_{i n}$ at the inlet of the channel, zero- pressure and viscous stresses at the outlet, no-slip and no-penetration conditions are imposed at the channel walls. To complete the set of conditions, a prescribed temperature of $T_{\text {in }}$ of $300 \mathrm{~K}$, and conditions of continuity of both temperature; i.e., $T=T_{s}=$ $T_{w}$, and heat flux; i.e., $k_{f} \partial T / \partial n=k_{s} \partial T_{s} / \partial n=k_{s} \partial T_{w} / \partial n$, at the solid-fluid interface walls are also imposed. For the solid block, the heat-generating chip is modeled by imposing a uniform heat influx $q^{\prime \prime}=47 \mathrm{~W} / \mathrm{cm}^{2}$, at the bottom surface (on a $10 \mathrm{~mm} \times 1.5 \mathrm{~mm}$ area) with all other surface area boundaries of the solid being considered adiabatic.

\section{Numerical Technique and Grid Independence Tests}

Equations (3)-(6) were first discretized on the corresponding computational domains using the finite element method, and then solved with the COMSOL Multiphysics (http://www.comsol.com) software. The procedure used is as follows. For each geometry, the computational domains for the channel $\Omega_{f}$, and the block $\Omega_{s}$, are discretized using three-dimensional unstructured meshes. Four-node tetrahedral elements are used for the fluid temperature, velocity and pressure, and to calculate the temperatures within the solid structure. It is important to note that, since a sharp representation of the fluid-solid interface is necessary to enable accurate solutions in regions close to the walls, we apply a mesh comprised of hexahedral elements. The resulting system of algebraic equations is solved iteratively by the Generalized Minimum Residual (GMRES) solver. The relative tolerance for calculations of velocity, pressure and temperatures in the channel, and solid block, is $10^{-6}$. At the inlet of the channel-block model, a discontinuous Galerkin condition [16] is used to accurately setup the thermal 
condition for the fluid $\left(T_{i n}=\right.$ constant $)$ and the solid $\left(\partial T_{s} / \partial n=0\right)$ domains, and sharply distinguish between them at the fluid-solid interface.

To ensure grid independence of the numerical results, various grids sizes were assessed using different values of inlet velocity. Fig. 2 illustrates a typical set of convergence tests for the fluid pressure $p$, streamwise velocity $u$ and temperature $T$, at a fixed point in the domain $\Omega_{f}$. The model is a single wavelength configuration with hydraulic diameter $D_{h}=500 \mu \mathrm{m}, A=200 \mu \mathrm{m}$, and $R e=150$. The figure shows that the error values in $u$ and $p$ vary significantly in the range analysed (2.9 and 3.4 million elements), whereas those of $T$ are essentially constant. A mesh with 3.6 million elements,

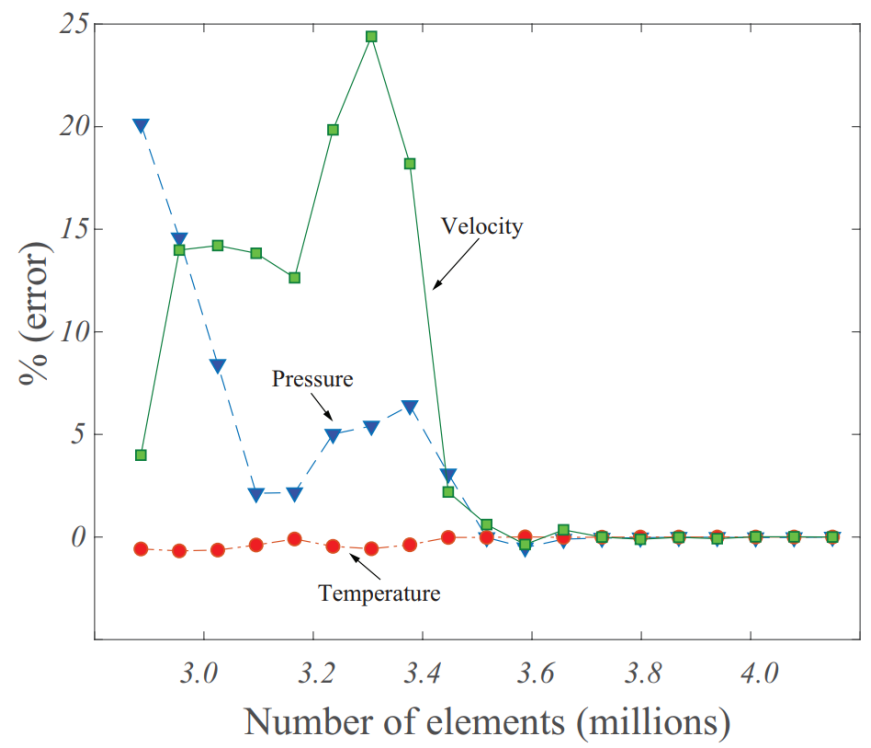

Fig. 2: Grid independence test-results.

In the case of the solid-block material, on the other hand, regardless of the type of material used (c.f. Table 1), a maximum number of only 282,000 elements are necessary to achieve grid independence of the solution; the error with respect to a grid three times as dense, is essentially zero. Thus for the models considered here, a total number of 3.6 million elements are used for the domain comprising both solid and fluid regions. Typical CPU times are less than 11 hours per run.

\section{Results and Analysis}

To analyse the impact of the material selected for the solid block upon the heat transfer enhancement in the single and multi-harmonic-wavy channel geometries, in addition to copper $(\mathrm{Cu})$, here we consider aluminium $(\mathrm{Al})$, and silicon $(\mathrm{Si})$. For each material, the properties corresponding to specific heat $c_{p, s}$, thermal conductivity $k_{s}$, and density $\rho_{s}$, at a reference temperature of $300 \mathrm{~K}$, are given in Table 1 . On the other hand, the fluid properties are those of water: $c_{p, f}=4.2 \mathrm{~kJ} / \mathrm{kg} \cdot \mathrm{K}$ is the specific heat, $k_{f}=0.6 \mathrm{~W} / \mathrm{m} \cdot \mathrm{K}$ is the thermal conductivity, $\mu=1.007 \times 10^{-3} \mathrm{~Pa} \cdot \mathrm{s}$ is the viscosity, and $\rho_{f}=$ $908.4 \mathrm{~kg} / \mathrm{m}^{3}$ is the density. Based on the densities from Table 1, the constant-volume solid domain equates to the amount of mass of each material as: $m_{A l}=1.08 \times 10^{-3} \mathrm{~kg}, m_{C u}=3.57 \times 10^{-3} \mathrm{~kg}$, and $m_{S i}=9.31 \times 10^{-4} \mathrm{~kg}$. 
Table 1: Properties of the solid-block material used in simulations.

\begin{tabular}{|c|c|c|c|}
\hline Material & Parameter & Description & Value \\
\hline \multirow{3}{*}{$\mathrm{Al}$} & $\mathrm{c}_{\mathrm{p}, \mathrm{s}}$ & Specific heat & $0.900[\mathrm{~kJ} / \mathrm{kg} \cdot \mathrm{K}]$ \\
& $\mathrm{k}_{\mathrm{s}}$ & Thermal conductivity & $205[\mathrm{~W} / \mathrm{m} \cdot \mathrm{K}]$ \\
& $\rho_{\mathrm{s}}$ & Solid density & $2700\left[\mathrm{~kg} / \mathrm{m}^{3}\right]$ \\
\hline \multirow{3}{*}{$\mathrm{Cu}$} & $\mathrm{c}_{\mathrm{p}, \mathrm{s}}$ & Specific heat & $0.375[\mathrm{~kJ} / \mathrm{kg} \cdot \mathrm{K}]$ \\
& $\mathrm{k}_{\mathrm{s}}$ & Thermal conductivity & $403.7[\mathrm{~W} / \mathrm{m} \cdot \mathrm{K}]$ \\
& $\rho_{\mathrm{s}}$ & Solid density & $8933\left[\mathrm{~kg} / \mathrm{m}^{3}\right]$ \\
\hline \multirow{2}{*}{$\mathrm{Si}$} & $\mathrm{c}_{\mathrm{p}, \mathrm{s}}$ & Specific heat & $0.71[\mathrm{~kJ} / \mathrm{kg} \cdot \mathrm{K}]$ \\
& $\mathrm{k}_{\mathrm{s}}$ & Thermal conductivity & $130[\mathrm{~W} / \mathrm{m} \cdot \mathrm{K}]$ \\
& $\rho_{\mathrm{s}}$ & Solid density & $2328[\mathrm{~kg} / \mathrm{m} 3]$ \\
\hline
\end{tabular}

In the case of the single-wave configuration, the constant cross-section channel is described as a lofted curve consisting of a sinusoidal region [c.f., Fig. 1(a)] modelled as $y(x)=A \cdot \sin (2 \pi x / \lambda)$ for $x \in[0,16] \mathrm{mm}$, with $x^{*}=x+2 \mathrm{~mm}$ (i.e., $x \in$ $[2,18] \mathrm{mm})$, and $z \in[-250,250] \mu \mathrm{m}$. $A$ is the wave amplitude and $\lambda$ is the wavelength of the wavy channel. For the harmonic wave geometries, secondary curves in the form of $(A /|n|) \cdot \sin (2 \pi x / \lambda)$, with $n= \pm 2$, are added in the region $x \in$ $[0,16] \mathrm{mm}$, to the fundamental wavy geometry, $y(x)$, as shown in Fig. 1(a). For all geometries, the governing equations (3)(6) were solved for $A=\{0,50,100,150,200\} \mu \mathrm{m}, R e=\{50,100,150\}$ with $R e=D_{h} u_{i n} \rho_{f} / \mu$ ( $D_{h}$ is the hydraulic diameter of the channel), $\lambda=2 \mathrm{~mm}$, and a heat influx $q^{\prime \prime}=47 \mathrm{~W} / \mathrm{cm}^{2}$, for the three materials considered.

Solutions of the governing equations are given in Figs. 3, 4 and 5, for the devices with the following block materials: aluminium $(A l)$, copper $(C u)$, and silicon $(S i)$, respectively. The figures show the temperature contours computed at the midplane $z=0$, for the straight, wavy, and representative harmonic $(n= \pm 2)$ channels, with a wave amplitude of $A=150 \mu \mathrm{m}$ and $R e=150$.

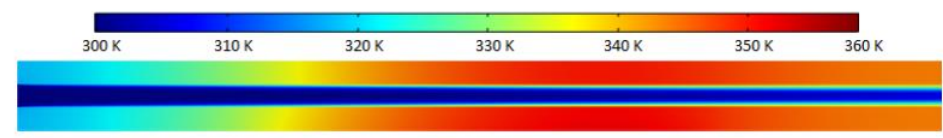

(a) straight: $A=0$.

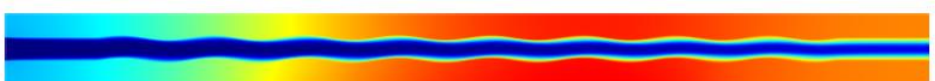

(b) wavy: $A=50 \mu \mathrm{m}$.

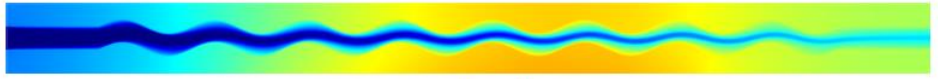

(d) wavy: $A=150 \mu \mathrm{m}$.

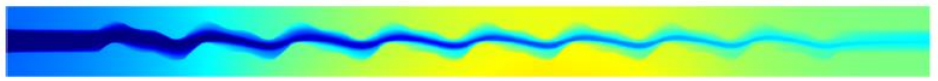

(f) harmonic: $A=150 \mu \mathrm{m}, n=+2$.

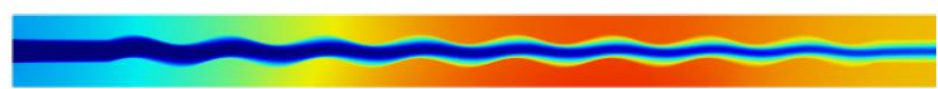

(c) wavy: $A=100 \mu \mathrm{m}$.

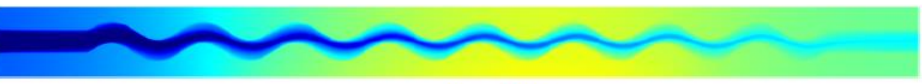

(e) wavy: $A=200 \mu \mathrm{m}$.

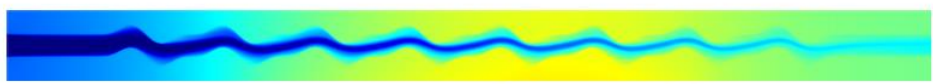

(g) harmonic: $A=150 \mu \mathrm{m}, n=-2$.

Fig. 3: Temperature contours for $A l$-material and $R e=150$. 


\begin{tabular}{lllllll}
\hline $300 \mathrm{~K}$ & $310 \mathrm{~K}$ & $320 \mathrm{~K}$ & $330 \mathrm{~K}$ & $340 \mathrm{~K}$ & $350 \mathrm{~K}$ & $360 \mathrm{~K}$ \\
\hline
\end{tabular}

(a) straight: $A=0$.

(b) wavy: $A=50 \mu \mathrm{m}$.

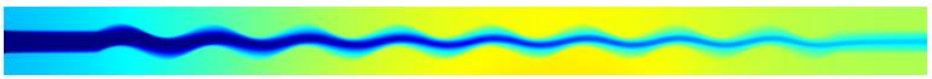

(d) wavy: $A=150 \mu \mathrm{m}$.

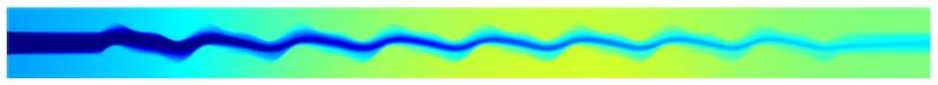

(f) harmonic: $A=150 \mu \mathrm{m}, n=+2$.

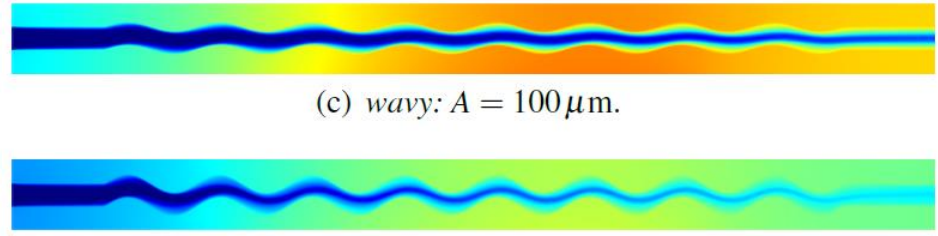

(e) wavy: $A=200 \mu \mathrm{m}$.

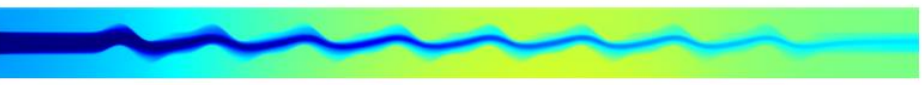

(g) harmonic: $A=150 \mu \mathrm{m}, n=-2$.

Fig. 4: Temperature contours for $C u$-material and $R e=150$.

\begin{tabular}{lllllll}
\hline $300 \mathrm{~K}$ & $310 \mathrm{~K}$ & $320 \mathrm{~K}$ & $330 \mathrm{~K}$ & $340 \mathrm{~K}$ & $350 \mathrm{~K}$ & $360 \mathrm{~K}$ \\
\hline & & & & & \\
\hline
\end{tabular}

(a) straight: $A=0$.

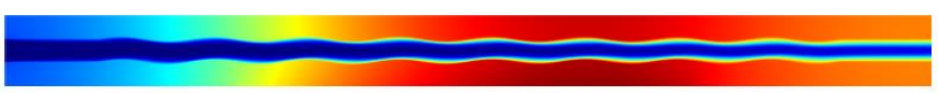

(b) wavy: $A=50 \mu \mathrm{m}$.

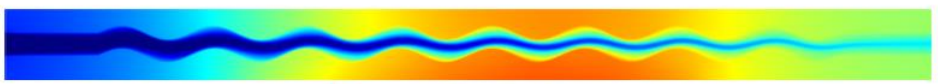

(d) wavy: $A=150 \mu \mathrm{m}$.

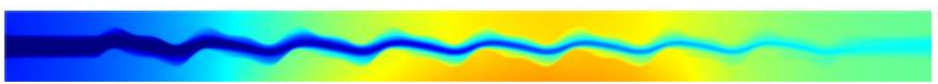

(f) harmonic: $A=150 \mu \mathrm{m}, n=+2$.

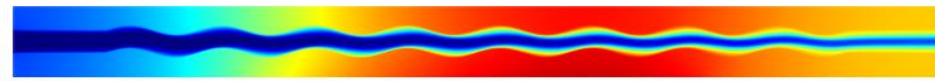

(c) wavy: $A=100 \mu \mathrm{m}$.

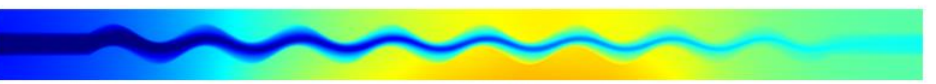

(e) wavy: $A=200 \mu \mathrm{m}$.

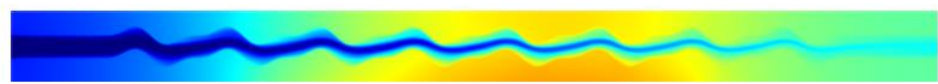

(g) harmonic: $A=150 \mu \mathrm{m}, n=-2$.

Fig. 5: Temperature contours for $S i$-material and $R e=150$.

From the figures, some common features can be identified. First, regardless of the material, as the amplitude $A$ increases, so does the effectiveness of the device in transfer energy as the temperatures of the block decrease proportionally. This correlates with the increase in the water temperatures indicating larger advection of heat. Second, the harmonic configurations are much more effective than the single-wave ones; e.g., the decrease in block temperatures, along the streamwise direction, is much higher in the harmonic channels with a smaller amplitude of $A=150 \mu \mathrm{m}$, than that of the single wave with a higher amplitude of $A=200 \mu \mathrm{m}$. This is also noticed as larger inlet-to-outlet temperature differences are present in these configurations. On the other hand, the effect of block material is seen as the temperatures in those made of copper and aluminium, which have higher thermal conductivities, have lower - and more uniform values than those of channels made of silicon.

By taking the wavy configuration as example, with those of the harmonic geometries being qualitatively similar, the corresponding results for the pressure drop $\Delta p$, as a function of both $A$ and $R e$, are given in Fig. 6, the results for the heat transfer cases being shown in Fig. 7. From Fig. 6, it can be observed that, as expected, the pressure-drop values in 
the channels increase with amplitude and Reynolds number, but they are also independent of the solid-block selection. Such decoupling of $\Delta p$ with respect to the type of material chosen for the block is primarily due to the assumptions of constant fluid (and block) properties; i.e., properties are independent of temperature, which were made here. On the other hand, from Fig. 7(a), which shows the overall change in water temperature between the channel inlet and outlet, $\Delta \bar{T}=\Delta \bar{T}_{\text {out }}-\Delta \bar{T}_{\text {in }}$, for $R e=\{50,100,150\}, A=\{0,50,100,150,200\} \mu \mathrm{m}$, and the three representative solid block materials, it can be seen that for all values of $R e$ and $A$, the largest fluid temperature changes occur for the channel with the $S i$-block, followed closely by those with the $A l$ - and $C u$-blocks, respectively. The effect of $R e$ in the fluid temperature-changes is also noticeable, as the values decrease with increasing $R e$ number; i.e., the $\Delta \bar{T}$-values for $R e=50$ are roughly twice as those for $R e=100$, and three times as those of $R e=150$. This trend is expected due to the constancy in the overall heat removal is constant and the inlet-to-outlet energy balance for the fluid inside the channel. The aforementioned behaviour is confirmed in Fig. 7(b), showing that the value of the overall-surface-average wall temperature of the channel block interface, $\bar{T}_{w}$, for the three block materials, decreases with increasing $A$, and $R e$, and correlates with the results for $\Delta \bar{T}$.

As was the case in the work of Moon et al. [1], and Grajeda et al. [17], in the present study the heat transfer solutions are quantified with the surface-averaged Nusselt number $\mathrm{Nu}$, defined as

$$
N u=\frac{h_{m} D_{h}}{k_{f}}
$$

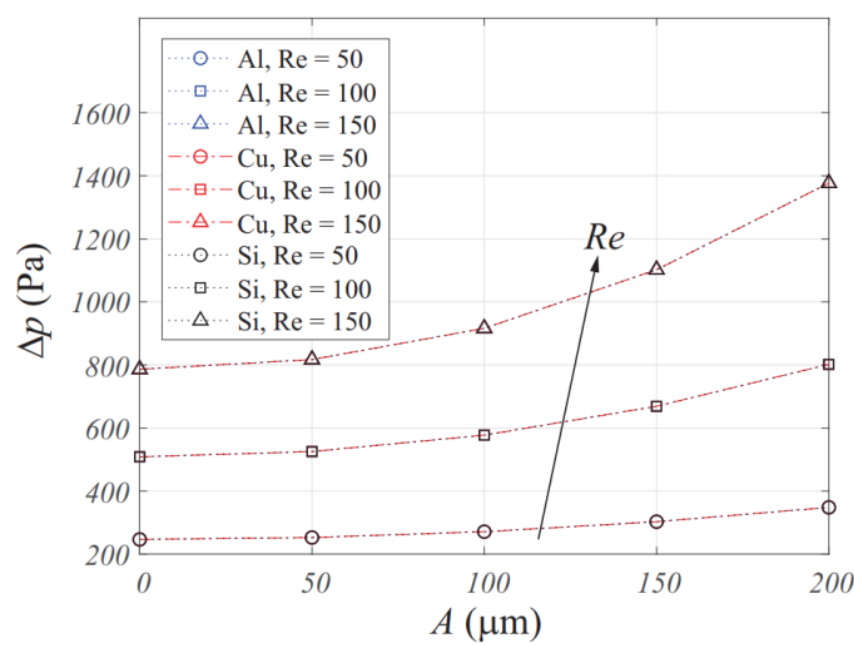

Fig. 6: Variation of fluid pressure drop with amplitude and Reynolds number. 


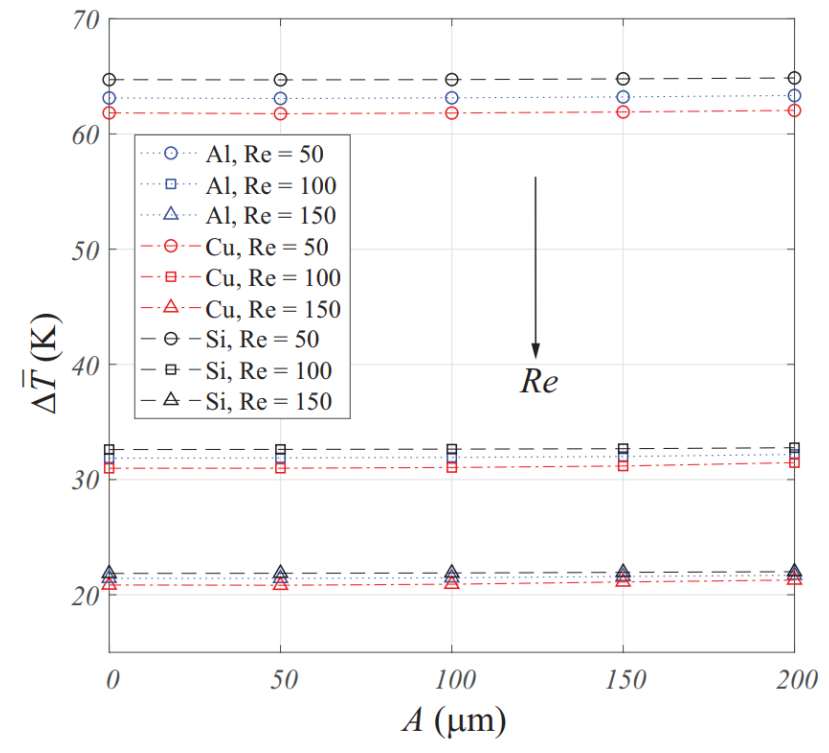

(a) Inlet-outlet change in water temperature.

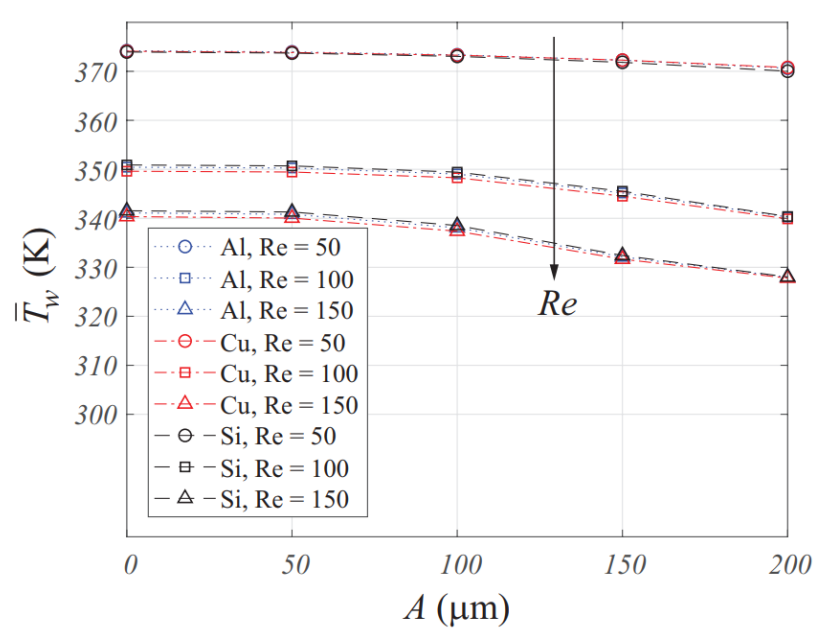

(b) Surface-average wall temperature.

Fig. 7: Fluid and block temperatures in the wavy channel configuration.

where $h_{m}$ is the average heat transfer coefficient, given as

$$
h_{m}=\frac{Q}{A_{w}\left(T_{w}-T_{m}\right)}
$$

where $A_{w}$ is the heat transfer surface between fluid and the solid block, $T_{w}$ and $T_{m}$, are the average temperature of the channel wall and the average bulk temperature of the cooling water $T_{m}=\left(T_{\text {in }}+\bar{T}_{\text {out }}\right) / 2$. On the other hand, $Q=q^{\prime \prime} A_{\text {base }}$, is the total heat load generated by the chip, $q^{\prime \prime}$ is heat influx, and $A_{\text {base }}$ is the bottom surface area upon which the constant- $q^{\prime \prime}$ boundary condition is applied.

Figure 8 shows the values of the average Nusselt number $N u$ - with Fig. 8(a) illustrating the results for the wavy channels and Fig. 8(b) displaying those for the harmonic: $n= \pm 2$ channels - as functions of the amplitude $(A=$ $\{0,50,100,150,200\} \mu \mathrm{m})$, and Reynolds number $(R e=\{50,100,150\})$, for the $\mathrm{Si}$-, $\mathrm{Al}$ - and $\mathrm{Cu}$-channel-block materials. From both subfigures it can be noticed that, although there are differences among the values of $N u$ for the three different block materials, these are constrained to less than $3 \%$ and therefore insignificant, indicating that due to consistently high thermal conductivity of the materials, the impact of the block-materials considered, on the Nusselt number for the fluid, is negligible. This is consistent with the results presented in Figs. 7(a) and 7(b), for both the inletto-outlet overall change in water temperature $\Delta \bar{T}$ and the overall-surface-average wall temperature of the channel block interface, $\bar{T}_{w}$. On the other hand, as demonstrated in [1], Fig. 8(b) shows that the inclusion of harmonic surfaces - in this case with $n= \pm 2$, which provided the best results - with all conditions (e.g., $R e=150$ and $A=150 \mu \mathrm{m}$ ) held constant, substantially enhances the transfer of energy by the device. It is to note that the general trends in the results for the harmonic geometries are the same to those shown here for the wavy configurations. 


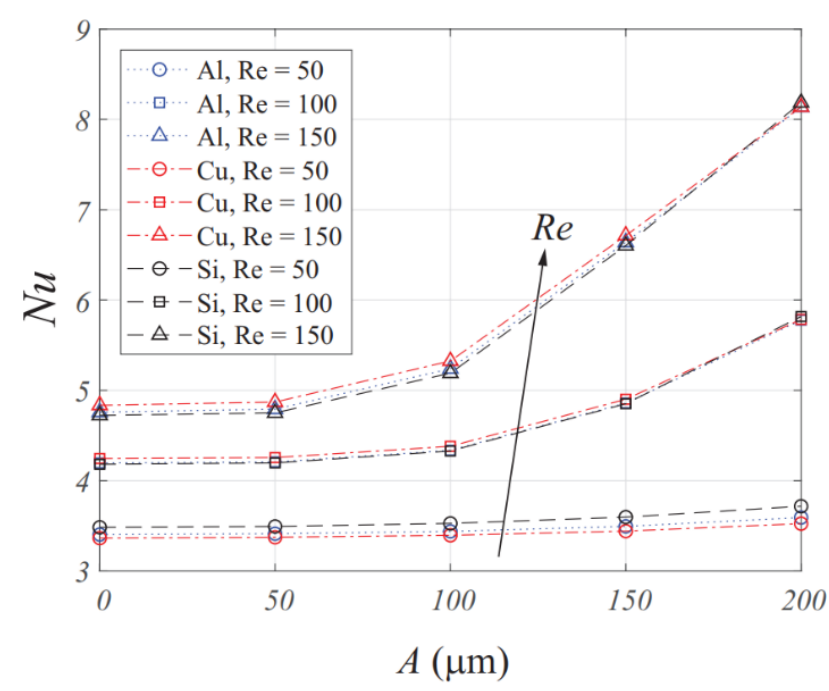

(a) Wavy channel configuration.

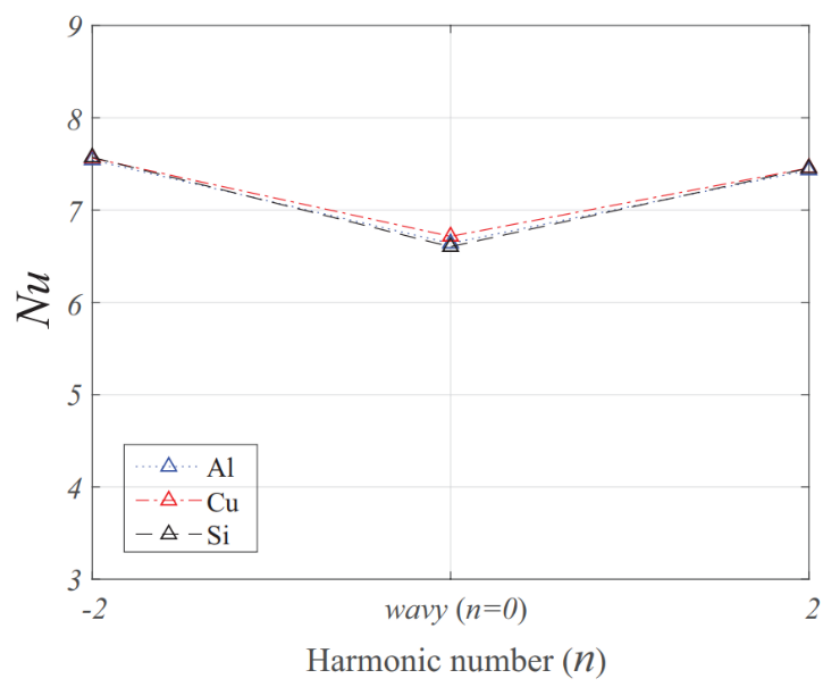

(b) Harmonic: $n \pm 2$, channel geometry; $A=150 \mu \mathrm{m}, R e=150$.

Fig. 8: Average Nusselt number for wavy and harmonic configurations.

The effectiveness of the device is analysed by defining the performance factor $P F$, as the ratio of the Nusselt number $\mathrm{Nu}$ of the wavy/harmonic channel to that of a straight channel, divided by the cube-root of the ratio of the pressure drop of the wavy/harmonic channel to the straight channel, as

$$
P F=\frac{N u_{w v} / N u_{s t}}{\left(\Delta p_{w v} / \Delta p_{s t}\right)^{1 / 3}}
$$

The solutions of $P F$ are given in Fig. 9 as functions of both amplitude $A$, and Reynolds number $R e$, for $R e=50,100$ and 150. Again, the wavy configuration is included as example, with the harmonic geometries having the same type of behaviour, but also underperforming the wavy device as reported in [1]. From the figure, it can be seen for small values of $R e$ (e.g., $R e=50$ ); but as $R e$ increases to 100 , and 150 , the values of $P F$ substantially increase, indicating a larger enhancement in the transfer of heat relative to the increased pumping power required, thus larger effectiveness. 


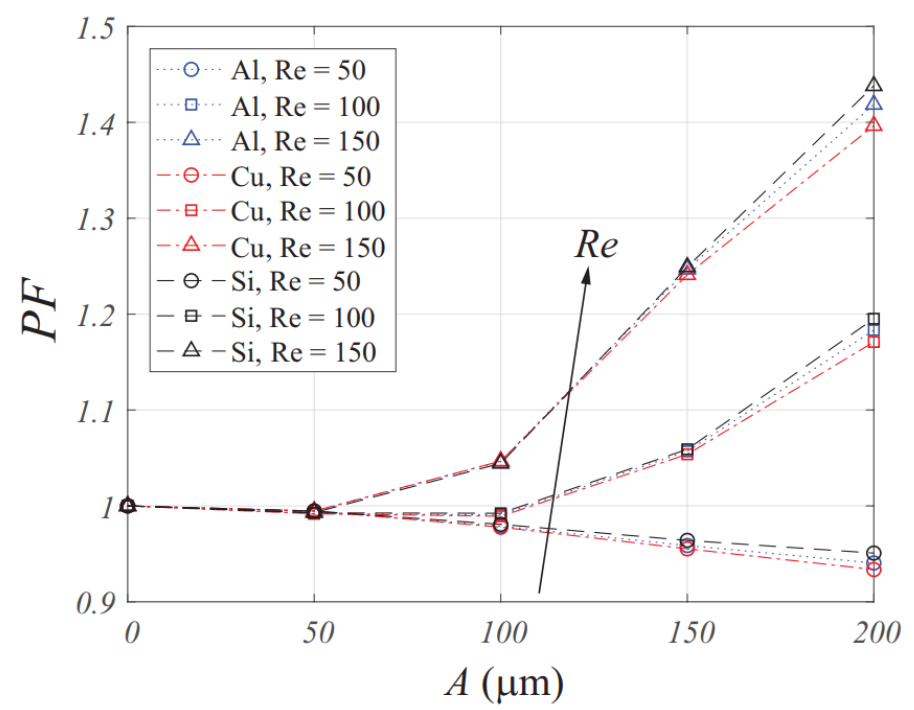

Fig. 9: Performance factor vs. amplitude.

\section{Conclusion}

Continuous development of compact electronic devices requires cooling systems with larger heat removal capabilities, and single-phase fluid-flow micro-channels with wavy configurations offer an avenue to improve their thermal management. In our previous work [1], the enhancement of heat transfer performance, at the cost of a slightly larger pressure drop, in microchannels has been recently analysed using multi-harmonic surface topologies with promising results. In the present work, we have studied numerically the effects of material selection on the heat transfer enhancement in these types of microchannels under different geometric parameters and operating conditions to remove a constant heat influx. The materials chosen are copper, aluminium and silicon. Detailed numerical simulations of the single-wavy- and multi-harmonic-channels have shown that: (a) regardless of the material, as wave amplitude and Reynolds number increase, so does the effectiveness of the device. (b) The harmonic geometries are more effective than the single-wave layouts. (c) The impact of block material is high in the diffusion of heat occurring in the solid block surrounding the channel, with copper and aluminium being the most effective in enabling lower block temperatures as compared to those obtained with channel-blocks fabricated with silicon. (d) The effect of material selection on the Nusselt number for the fluid, however, is negligible. (e) Finally, the effectiveness of the device, via the performance factor $P F$, is negative for small values of Reynolds number, but it improves substantially at larger $R e$-values $(R e>100)$, indicating a larger heat transfer enhancement relative to the required pumping power required, thus larger effectiveness.

\section{Acknowledgements}

Justin Moon is the recipient of a CEaS fellowship for which we are grateful. This work has been supported by the National Science Foundation under Award No. HRD-1547723.

\section{References}

[1] J. Moon, J. R. Pacheco, and A. Pacheco-Vega, "Heat transfer enhancement in wavy micro-channels through multiharmonic surfaces," in Proceedings of the ASME 2018 International Mechanical Engineering Congress and Exposition, Pittsburgh, PA, USA, 2018. IMECE2018-86425.

[2] D. B. Tuckerman and R.F.W. Pease. High-performance heat sinking for VLSI. Electron Device Letters, IEEE, vol. 2, no. 5, pp. 126-129, 1981.

[3] P. S. Lee, S. V. Garimella, and D. Liu, "Investigation of heat transfer in rectangular microchannels," Int. J. Heat Mass Transfer, vol. 48, pp. 1688-1704, 2005. 
[4] X. L. Xie, Z. J. Liu, Y. L. He, and W. Q. Tao, "Numerical study of laminar heat transfer and pressure drop characteristics in a water-cooled minichannel heat sink," Applied Thermal Engineering, vol. 29, pp. 64-74, 2009.

[5] T. M. Harms, M.J. Kazmierczak, and F. M. Gerner, "Developing convective heat transfer in deep rectangular microchannels," Int. J. Heat and Fluid Flow, vol. 20, pp. 149-157, 1999.

[6] W. Qu and I. Mudawar, "Experimental and numerical study of pressure drop and heat transfer in a single-phase microchannel heat sink," Int. J. Heat Mass Transfer, vol. 45, pp. 2549-2565, 2002.

[7] S. Sasaki and T. Kishimoto, "Optimal structure for microgrooved cooling fin for high-power LSI devices," Electronics Letters, vol. 22, pp. 1332-1334, 1986.

[8] Y. Sui, C. J. Teo, P. S. Lee, Y. T. Chew, and C. Shu, "Fluid flow and heat transfer in wavy micro channels," Int. J. Heat Mass Transfer, vol. 53, pp. 2760-2772, 2010.

[9] Y. Sui, P. S. Lee, and C. J. Teo, "An experimental study of flow friction and heat transfer in wavy microchannels with rectangular cross section," Int. J. Thermal Sciences, vol. 50, pp. 2473-2482, 2011.

[10] L. Gong, K. Kota, W. Tao, and Y. Joshi, "Parametric numerical study of flow and heat transfer in microchannels with wavy walls," ASME J. Heat Transfer, vol. 133, no. 5, 2011. Paper No. 051702.

[11] K. Vafai and L. Zhu, "Analysis of two-layered micro-channel heat sink concept in electronic cooling," Int. J. Heat Mass Transfer, vol. 42, pp. 2287-2297, 1999.

[12] G. N. Xie, Z. Y. Chen, B. Sunden, and W. H. Zhang, "Numerical predictions of the flow and thermal performance of water-cooled single-layer and double-layer wavy microchannel heat sinks," Numerical. Heat Transfer, Part A, vol. 63, pp. 201-225, 2013.

[13] G. N. Xie, J. Liu, Y. Liu, B. Sunden, and W. H. Zhang, "Comparative study of thermal performance of longitudinal and transversal-wavy microchannel heat sinks for electronic cooling," ASME J. Electronic Packaging, vol. 135, no. 2, 2013. Paper No. 021008.

[14] Q. Mao and P. Deng, "Study of micro-channel shape and material property on cooling performance of high power LED chips," in 9th IEEE International Conference on Nano/Micro Engineered and Molecular Systems (NEMS), pages 498-501, Hawaii, USA, 2014.

[15] A. Uday Kumar, A. Javed, and S. K. Dubey, "Material selection for microchannel heatsink: Conjugate heat transfer simulation," IOP Conference Series: Materials Science and Engineering, vol. 346, no. 1, pp. 012024, 2018.

[16] D. N. Arnold, F. Brezzi, B. Cockburn, and L. D. Marini, "Unified analysis of discontinuous Galerkin methods for elliptic problems," SIAM Journal of Numerical Analysis, vol. 39, pp. 1749-1779, 2002.

[17] E. Grajeda, C. Gaunthier, F. Dugast, J. R. Pacheco, and A. Pacheco-Vega, "Conjugate heat transfer analysis of multiharmonic wavy mico-channels," in Proceedings of the International Conference on Energy Systems, Istanbul, Turkey, 2015, pp. 1-8. 